BMJ Open

Diabetes

Research

\& Care

\title{
Huntingtin-associated protein 1 plays an essential role in the pathogenesis of type 2 diabetes by regulating the translocation of GLUT4 in mouse adipocytes
}

\author{
Yan-Ju Gong, ${ }^{1}$ Ying Feng,,${ }^{1}$ Yuan-Yuan Cao, ${ }^{1}$ Jia Zhao, ${ }^{1}$ Wei Wu, ${ }^{2}$ Ya-Yun Zheng, ${ }^{1}$ \\ Jia-Rui Wu, ${ }^{1}$ Xin Li, ${ }^{3}$ Gui-Zhi Yang, ${ }^{1}$ Xue Zhou (D) ${ }^{1}$
}

To cite: Gong Y-J, Feng Y, Cao Y-Y, et al. Huntingtinassociated protein 1 plays an essential role in the pathogenesis of type 2 diabetes by regulating the translocation of GLUT4 in mouse adipocytes. BMJ Open Diab Res Care 2020;8:e001199. doi:10.1136/ bmjdrc-2020-001199

- Supplemental material is published online only. To view please visit the journal online (http://dx.doi.org/10.1136/ bmjdrc-2020-001199).

Y-JG and YF contributed equally.

G-ZY and XZ contributed equally.

Received 15 January 2020 Revised 30 July 2020 Accepted 5 September 2020

Check for updates

C Author(s) (or their employer(s)) 2020. Re-use permitted under CC BY-NC. No commercial re-use. See rights and permissions. Published by BMJ.

For numbered affiliations see end of article.

Correspondence to Professor Xue Zhou; zhouxue7239@163.com and Professor Gui-Zhi Yang; ygzxky@163.com

\section{ABSTRACT}

Objective Glucose disposal by insulin-responsive tissues maintains the body glucose homeostasis and insulin resistance leads to a risk of developing type 2 diabetes (T2DM). Insulin stimulates the translocation of glucose transporter isoform 4 (GLUT4) vesicles from intracellular compartments to the plasma membrane to facilitate glucose uptake. However, the underlying mechanisms of GLUT4 vesicle translocation are not well defined. Here we show the role of huntingtin-associated protein 1 (HAP1) in GLUT4 translocation in adipocytes and the pathogenesis of T2DM.

Research design and methods The parameters for glucose metabolism including body weight, glucose tolerance and insulin tolerance were assessed in wildtype (WT) and $\mathrm{Hap1}^{+/-}$mice. HAP1 protein expression was verified in adipose tissue. Hap1 mRNA and protein expression was monitored in adipose tissue of high-fat diet (HFD)-induced diabetic mice. Insulin-stimulated GLUT4 vesicle translocation and glucose uptake were detected using immunofluorescence techniques and quantified in primary adipocytes from $\mathrm{Hap}^{-1-}$ mice. The interaction between HAP1 and GLUT4 was assessed by immunofluorescence colocalization and co-immunoprecipitation in HEK293 cells and adipose tissue. The role of sortilin in HAP1 and GLUT4 interaction was approved by co-immunoprecipitation and RNA interference.

Results The expression of Hap1 mRNA and protein was detected in WT mouse adipose tissue and downregulated in adipose tissue of HFD-induced diabetic mice. Hap $1^{+/-}$ mice exhibited increased body weight, pronounced glucose tolerance and significant insulin intolerance compared with the WT mice. HAP1 colocalized with GLUT4 in mouse adipocytes and cotransfected HEK293 cells. Furthermore, the insulin-stimulated GLUT4 vesicle translocation and glucose uptake were defective in $\mathrm{Hap}^{-1-}$ adipocytes. Finally, sortilin mediated the interaction of HAP1 and GLUT4.

Conclusions Our study showed that HAP1 formed a protein complex with GLUT4 and sortilin, and played a critical role in insulin-stimulated GLUT4 translocation in adipocytes. Its downregulation may contribute to the pathogenesis of diabetes.

\section{Significance of this study}

What is already known about this subject?

- Insulin-stimulated glucose transporter isoform 4 (GLUT4) translocation needs the help of transporters and regulators.

- Huntingtin-associated protein 1 (HAP1) highly expresses in nervous and endocrine systems.

- HAP1 acts as an accessory molecule for molecular motors.

What are the new findings?

- HAP1 is expressed in adipocytes.

- HAP1 forms a protein complex with GLUT4 and sortilin and participates in GLUT4 translocation.

- Hap1 mRNA and protein is downregulated in diabetic state.

How might these results change the focus of research or clinical practice?

- In addition to neurons and endocrine cells, HAP1 also participates in the physiological functions of other cells and plays a broader role.

- HAP1 may be a potential therapeutic target for type 2 diabetes mellitus.

\section{INTRODUCTION}

The hallmark of type 2 diabetes mellitus (T2DM) pathogenesis is insulin resistance, which includes a decrease in target cell's metabolic response to insulin and an impaired lowering effect of insulin on blood glucose. ${ }^{1}$ Accumulated evidences suggest that multiple mechanisms are responsible for the decrease of insulin-stimulated glucose utilization and the development of insulin resistance, including genetic mutations, posttranslational modifications, protein-protein interaction and protein phosphorylationdephosphorylation events, and formation of inhibitory complexes. ${ }^{2}$ 
Glucose transporter isoform 4 (solute carrier family 2 , facilitated glucose transporter member 4, GLUT4) is the main insulin-sensitive glucose transporter primarily expressed in adipose tissue and striated muscle, and less expressed in liver, hippocampus and other tissues. ${ }^{34}$ The abnormal expression of GLUT4 and the impaired intracellular transport are responsible for the occurrence of insulin resistance. ${ }^{5}$ Moreover, the mRNA and protein levels of GLUT4 are downregulated in the adipose tissue of T2DM. ${ }^{67}$ In response to insulin stimulation, GLUT4 storage vesicles (GSVs) first translocate and then fuse with plasma membrane. This process is precisely regulated and involves various molecules. ${ }^{8}$ However, the exact mechanism is unclear. Thus, identification of molecules involved in GLUT4 translocation will help us clarify the glucose uptake process in cells and shed new light to the pathogenesis of insulin resistance.

Recent studies have shown that the interacting proteins of huntingtin-associated protein 1 (HAP1) in neural cells, such as sortilin, kinesin family motor protein 5 (KIF5B) and myosin 5 (MYO5), ${ }^{9-11}$ help with GLUT4 translocation in adipocyte. ${ }^{12-16}$ HAP1 is one of the components of molecular motors, mainly expressed in the central nervous system, ${ }^{17}{ }^{18}$ as well as in endocrine organs secreting nitrogenous hormone. ${ }^{19}$ HAP1 has been found to interact with microtubules and various membranous organelles (eg, mitochondria, endoplasmic reticulum, intracellular bodies and lysosomes). ${ }^{20}$ It also participates in intracellular transport of proteins such as androgen receptor, TATA-binding protein and gamma-aminobutyric acid (GABA) type A receptors, thus exerting different physiological regulatory roles in nervous and endocrine systems. ${ }^{21-25}$

Adipose tissue has been considered as an energy storage organ traditionally. Recent studies have suggested that adipose tissue functions as an endocrine organ and secretes nitrogenous hormones such as leptin, adiponectin and resistin. ${ }^{26-28}$ Despite that HAP1 is highly expressed in endocrine organs which secret nitrogenous hormones, the expression of this molecule in adipose tissue is unknown. Based on the distribution and function of HAP1, we hypothesized that HAP1 could be expressed in adipose tissue and involved in the insulin-stimulated GLUT4 translocation. The aberrant expression of HAP1 could disrupt the transport of GLUT4 and result into impaired glucose uptake, insulin resistance afterwards and finally contribute to the onset of T2DM. In this study, we investigate the expression of HAP1 in adipose tissue under diabetic state, the relationship between HAP1 and GLUT4 translocation in adipocytes and the consequence of HAP1 deficiency on the pathogenesis of T2DM.

\section{MATERIALS AND METHODS}

\section{Animals}

All experiments were performed in accordance with the guidelines on the care and use of animals for research purposes by the Institutional Animal Care and Use
Committee of Sichuan University, and all the procedures were approved by the Institutional Animal Care and Use Committee of Sichuan University.

The animals were maintained in an air-conditioned facility (temperature controlled at $25^{\circ} \mathrm{C} \pm 1^{\circ} \mathrm{C}$ ) with a 12-hour light/dark cycle with free access to water and standard rodent chow. C57BL/6J male mice were purchased from Jianyang Dashuo Animal Technology (Sichuan, China), and Hapl knockout male mice on a C57BL/6J genetic background $\left(\mathrm{Hap}^{\mathrm{tm} 1 \mathrm{Xjl}}\right)$ were purchased from the Jackson Laboratory (007749; Bar Harbor, Maine, USA). PCR genotyping of Hapl knockout mice was carried out using primers 5'-TTTTGGAG GTCTGGTCTCGCTCTG-3' / 5'-CGTCTTCCATCTTAGT GCGTTCAC-3' for wild type (WT) and 5'-TTTTGGAG GTCTGGTCTCGCTCTG-3' / $5^{\prime}$-CTTCATGTGGATGCTA GGGATCC-3' for knockout animals. The PCR identification results were partly showed in online supplemental figure S1.

For studies of diet-induced T2DM in mice, the WT mice were divided into two groups: the mice were fed with either normal diet (ND $\left(\mathrm{H}_{2} \mathrm{O} \leq 10 \%\right.$, protein $\geq 18 \%$, fat $\geq 4 \%$, fiber $\leq 5 \%$, ash content $\leq 8 \%$ ); Jianyang Dashuo Animal Technology, Sichuan, China) or high-fat diet (HFD $(60 \%$ ND, $20 \%$ sugar, $10 \%$ fat from lard, $10 \%$ chicken egg yolk); Experimental Animal Center of Sichuan University, Sichuan, China). HFD was introduced on the 13th week after birth. Metabolic parameters were monitored during the in vivo experiments.

\section{Body weight measurements}

The body weight of each animal was monitored and recorded using an electronic balance every 2 weeks from the age of 6 weeks.

\section{Metabolic measurements}

Glucose tolerance tests (GTTs) and insulin tolerance tests (ITTs) were performed as previously described ${ }^{29}$ Briefly, GTTs were conducted on mice fasted for 16 hours. The mice were injected intraperitoneally with glucose in $0.9 \%$ saline $(2 \mathrm{~g} / \mathrm{kg})$. ITTs were conducted on mice fasted for 4 hours. The mice were injected intraperitoneally with human insulin in $0.9 \%$ saline $(0.2 \mathrm{U} / \mathrm{kg})$. Blood glucose levels $(\mathrm{mmol} / \mathrm{L})$ were measured from tail vein blood using the Sannuo blood glucose monitor (Sinocare, Hunan, China) at indicated time points. Plasma insulin concentrations were measured by ELISA (Shanghai Elisa Biotech, Shanghai, China).

\section{Culture of fibroblast-derived adipocytes}

Mouse primary fibroblasts were isolated from back skin of newborn mice and cultured in standard medium (Dulbecco's Modified Eagle Medium (DMEM) supplemented with $100 \mu \mathrm{g} / \mathrm{mL}$ penicillin/streptomycin, $10 \%$ fetal bovine serum) (details showed in online supplemental file).

Differentiation of adipocytes derived from mouse primary fibroblasts was performed according to the 
manufacturer's protocol (Cyagen, Guangzhou, China). Briefly, the fibroblastswere seeded ata density of $2 \times 10^{4} \mathrm{cell} /$ $\mathrm{cm}^{2}$ in 6-well plates in standard medium. Two days after confluence, the medium was replaced with adipogenic differentiation medium A containing insulin (1:500), dexamethasone (1:1000), 3-isobutyl-1-methylxanthine $(1: 1000)$ and rosiglitazone (1:1000). After day 3, the medium A was replaced with adipogenic differentiation medium B containing only insulin (1:500) and the cells were cultured for at least 2 days before use (differentiation images shown in online supplemental figure S2).

For GLUT4 translocation assay, adipocytes were cultured in standard medium (DMEM supplemented with $100 \mu \mathrm{g} / \mathrm{mL}$ penicillin/streptomycin, $10 \%$ fetal bovine serum) for 24 hours and then serum starved in Krebs-Ringer buffer containing $0.2 \% \quad(\mathrm{w} / \mathrm{v})$ bovine serum albumin (BSA) for 2 hours followed by incubation with or without $100 \mathrm{nM}$ insulin for $30 \mathrm{~min}$. The cells were either fixed with $3.7 \%(\mathrm{w} / \mathrm{v})$ formaldehyde in phosphatebuffered saline (PBS) for immunofluorescence staining or harvested for isolation of cell membrane fraction using the Plasma Membrane Protein Isolation Kit (Bestbio, Shanghai, China) according to manufacturer's instructions (details shown in online supplemental file).

\section{Transfection of cell lines}

HEK293 cells were obtained from the American Type Culture Collection (Manassas, Virginia, USA). HEK293 cells were maintained in DMEM supplemented with $10 \%$ fetal bovine serum. To analyze the interaction of HAP1 and GLUT4, HEK293 cells were transiently cotransfected with HAP1-CFP (encoding HAP1 isoform A) and GLUT4mCherry plasmids with Lipofectamine 2000 (Invitrogen, Carlsbad, California, USA) according to manufacturer's instructions. To study the role of sortilin, HEK293 cells were cotransfected with the above two plasmids and sortilin-small interfering RNA (siRNA) (5'-GAGGCATTGTCTATTCCAA-3'). Forty-eighthours after transfection, the cells were used for fluorescence observation or co-immunoprecipitation (Co-IP) assay.

\section{Co-IP assay}

Co-IP was performed as described previously. ${ }^{30}$ Briefly, cell lysates were prepared from white adipose tissue from mouse epididymal adipose pad or HEK293 cells. The lysates were pre-cleared by adding protein $\mathrm{A} / \mathrm{G}$ magnetic beads (Bimake, Houston, Texas, USA) in lysis buffer, the mixture was rotated for $30 \mathrm{~min}$ in $4^{\circ} \mathrm{C}$ and then magnetic beads separated; the supernatant were kept for Co-IP experiment. The pre-cleared lysates $(500 \mu \mathrm{g})$ were incubated with $1 \mu \mathrm{g}$ of goat anti-HAP1 antibody (sc-12556; Santa Cruz, Dallas, Texas, USA) or goat anti-GLUT4 antibody (sc-1608, Santa Cruz) or rabbit anti-GLUT4 antibody (ab654; Abcam, Cambridge, UK) or rabbit antisortilin antibody (ab16640, Abcam) at $4^{\circ} \mathrm{C}$ for 12 hours, and then incubated with protein $\mathrm{A} / \mathrm{G}$ beads for 1 hour. The beads were washed five times with the lysis buffer and boiled in loading buffer for western blot. For negative control, goat and rabbit IgG and untransfected HEK293 were used for validation.

\section{Western blot}

Lysates of adipose and brain tissues were prepared using radioimmunoprecipitation assay lysis buffer (Beyotime, Jiangsu, China) containing protease inhibitors (SigmaAldrich, St. Louis, Missouri, USA). Ten microliters of the homogenates were kept for total protein level analysis using bicinchoninic acid (BCA) kits (Beyotime) according to the manufacturer's instructions. Tissue lysate, isolated cell membrane proteins and Co-IP samples were analyzed by $10 \%$ SDS-PAGE (sodium dodecyl sulfate-polyacrylamide gel electrophoresis) and transferred to polyvinylidene fluoride membrane. The membrane was first blocked in Tris-buffered saline containing $0.1 \%$ Tween-20 and incubated with appropriate primary (goat anti-HAP1 antibody (sc-12556, 1:1000; Santa Cruz), goat antiGLUT4 antibody (sc-1608, 1:1000; Santa Cruz), rabbit anti-GLUT4 antibody (ab654, 1:1000; Abcam), rabbit anti-sortilin antibody (ab16640, 1:1000; Abcam), rabbit anti-Na/K-ATPase antibody (3010, 1:1000; CST)) and secondary antibodies (rabbit anti-goat IgG-HRP antibody (sc-2768, 1:2000; Santa Cruz), goat anti-rabbit IgG-HRP antibody (sc-2004, 1:2000; Santa Cruz)). The membrane was developed with Immobilon Western (P90719; Millipore, Burlington, Massachusetts, USA) and examined via Bio-Rad ChemiDoc MP system (Bio-Rad, Hercules, California, USA). Captured images were quantified using ImageJ software (http://rsb.info.nih.gov/ij/).

\section{Quantitative real-time PCR (RT-qPCR)}

Total RNA was extracted using RNAiso Kit (Takara, Beijing, China) following the supplier's instructions. For each sample, $1 \mu \mathrm{g}$ of RNA was treated and reversetranscribed with PrimeScript RT reagent Kit with gDNA Eraser (Takara). SYBR Green RT PCR was performed using a CFX96 Real Time System (Bio-Rad) in triplicate using TB Green Premix Ex TaqII (Takara) with specific primers for mouse Hap1 (forward primer 5'-CGTGCGGCGTTTATTCGAG-3' and reverse primer 5'-GCTGTGTTCAGGTCCCGTTC- $3^{\prime}$ ) and the housekeeping gene $\beta$-actin (forward primer 5'-AGATTACTGCTCTGGC TCCTAGC-3' and reverse primer 5'-ACTCATCGTACT CCTGCTTGCT-3'). Gene of interest expression data were normalized by dividing the corresponding levels of $\beta$-actin for each sample.

\section{Immunostaining}

Immunofluorescence was performed with mouse anti-HAP1 antibody (NB110-74569, 1:1000; Novus, Littleton, Colorado, USA) and rabbit anti-GLUT4 antibody (ab654, 1:500; Abcam). Slices were incubated overnight with primary antibodies at $4^{\circ} \mathrm{C}$. DyLight 488-conjugated AffiniPure Goat Anti-rabbit secondary antibodies (115-485-205, 1:2000; Jackson ImmunoResearch, West Grove, Pennsylvania, USA) and DyLight 549-conjugated AffiniPure Goat Anti-mouse secondary 
antibodies (111-505-144, 1:2000; Jackson ImmunoResearch) were used for immunofluorescence staining, and the slices were then stained with DAPI $(5 \mu \mathrm{g} / \mathrm{mL})$. Images were captured using Zeiss LSM710 inverted confocal microscope.

The SPlink Detection Kits (ZSGB-BIO, Beijing, China) were used for immunohistochemistry staining and $\mathrm{DAB}$ was used as the chromogen. Digital images were captured using Precipoint software (Germany).

To compensate for the personal error, location of GLUT4 signal was classified into three categories: in the perinuclear region, diffused distribution in cytoplasm and on the cell membrane. GLUT4 distribution of each cell can fall into only one of these categories. In the light of GLUT4 translocation process, the priority order is membrane, cytoplasm and perinuclear region.

\section{Glucose uptake}

The glucose uptake was measured using a fluorescent D-glucose derivative, 2-NBDG (2-( $N$-(7-Nitrobenz-2-oxa1,3-diazol-4-yl)Amino)-2-Deoxyglucose), according to the published procedure. ${ }^{31}$ For fluorescent microplate reader measurement of 2-NBDG uptake level, $5 \times 10^{3}$ cells per well were seeded in 96-well black, clear-bottom culture plate (Corning, New York, USA). After overnight incubation, cells were cultured in serum-free low-glucose $(5.5 \mathrm{mM})$ DMEM for 4 hours and washed twice with serum-free glucose-free DMEM. Then cells were incubated in serum-free glucose-free DMEM supplemented with $100 \mathrm{nM}$ insulin and $100 \mu \mathrm{M}$ 2-NBDG for $30 \mathrm{~min}$. After incubation, cells were washed out of free 2-NBDG using PBS. The fluorescence retained in cell monolayers was measured with a fluorescence microplate reader (Thermo Fisher Scientific, Massachusetts, USA), set at an excitation wavelength of $485 \mathrm{~nm}$ and emission wavelength of $535 \mathrm{~nm}$. The representative 2-NBDG uptake images were captured using Leica DMI6000 inverted fluorescence microscope.

To detect glucose consumption, primary adipocytes were starved for 6 hours with serum-free DMEM, and then cultured in DMEM supplemented with 0.2\% BSA and $100 \mathrm{nM}$ insulin for 24 hours. Glucose oxidase method was applied to determine the glucose concentration in culture medium. ${ }^{32}$ Glucose consumption per hour of adipocytes was calculated by multiplying the glucose concentration difference in the supernatant before and after culturing by the reaction volume and then dividing the above product by the reaction time. To reduce the variation caused by the number of cells in each well, the total protein in each well was extracted and determined by BCA method. Thus, glucose consumption was presented as glucose consumption per $\mathrm{mg}$ protein per hour $(\mathrm{mmol} /(\mathrm{mg} \cdot$ hour $))$.

\section{Statistics}

GraphPad Prism V.7.00 for Windows (GraphPad Software, San Diego, California, USA) was used for standard statistical analysis of the data. Data were presented as mean \pm SEM. Simple pairwise comparisons were made using unpaired two-tailed t tests. Multiple comparisons were made using two-way analysis of variance (ANOVA) with Bonferroni post-tests. Comparison of proportion was evaluated by $\chi^{2}$ test. $\mathrm{P}$ value $<0.05$ was considered to be statistically significant.

\section{RESULTS}

\section{Hap1 mRNA and protein levels were downregulated in} adipose tissue of diabetic mice

We first examined HAP1 expression in adipose tissue with western blotting. Results showed that HAP1 was expressed in adipose tissue (figure 1A). HAP1 expression in adipocytes was confirmed with immunofluorescence and immunohistochemistry staining in an intracellular location and particularly in perinuclear compartment (figure 1B,C). The negative control was shown in online supplemental figure S3.

To investigate the expression of Hap1 under diabetic condition, HFD-induced diabetic model was applied. The glucometabolic parameters were monitored to confirm the model was successful (online supplemental figure S4). RT-qPCR analysis showed that mRNA expression of Hapl in adipose tissue was significantly lower in HFDfed group than in ND-fed group (figure 1D), and the protein levels of HAP1 also downregulated (figure 1E). The results suggested that HAP1 was reduced in adipose of HFD-fed mice and might take a part in the onset of diabetes.

\section{Reduction of Hap1 expression attenuated insulin-stimulated glucose uptake}

Since the homozygote mice $\left(\mathrm{Hapr}^{--}\right)$could not survive to adulthood, ${ }^{33}$ heterozygous mice $\left(\mathrm{Hapl}^{+/-}\right)$were used to investigate the impact of HAP1 on systemic glucose homeostasis, and glucometabolic parameters were assessed. We found $\mathrm{HapI}^{+/}$mice showed glycometabolism disorder and a greater risk of developing diabetic symptoms. First, the size and body weight of $\mathrm{Hapl}^{+/-}$ mice increased compared with WT mice (figure 2A). Despite $\mathrm{Hapl}^{+/-}$mice showed normal fasting glucose level (figure 2B), the plasma insulin level of $\mathrm{Hapl}^{+/-}$mice decreased (figure 2C). After intraperitoneal injection of glucose, the glucose tolerance curve in $\mathrm{HapI}^{+/-}$mice was different from that of WT mice. Hapl ${ }^{+/}$mice had larger glucose excursion range than WT mice (figure 2D). Meanwhile, $\mathrm{Hapl}^{+/-}$mice showed delayed glucose excursion after insulin stimulation and their blood glucose level could not return to the fasting level at $90 \mathrm{~min}$. Although the area under the insulin tolerance curves were similar, ITT results were significantly different between $\mathrm{Hapl}^{+/-}$and WT mice when analyzed by two-way ANOVA ( $\mathrm{p}<0.001)$, indicating $\mathrm{Hap}^{+/-}$mice had a delayed response to insulin stimulation (figure $2 \mathrm{E}$ ). Moreover, the glucose level decreased slower at $30 \mathrm{~min}$ in ITT in $\mathrm{Hapl}^{+/-}$mice than in WT mice (analyzed by t-test, $\mathrm{p}<0.05$ ) (figure 2E). The above results showed that $\mathrm{Hapl}^{+/-}$mice 


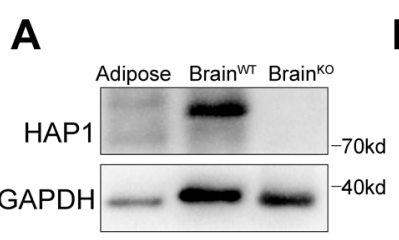

B
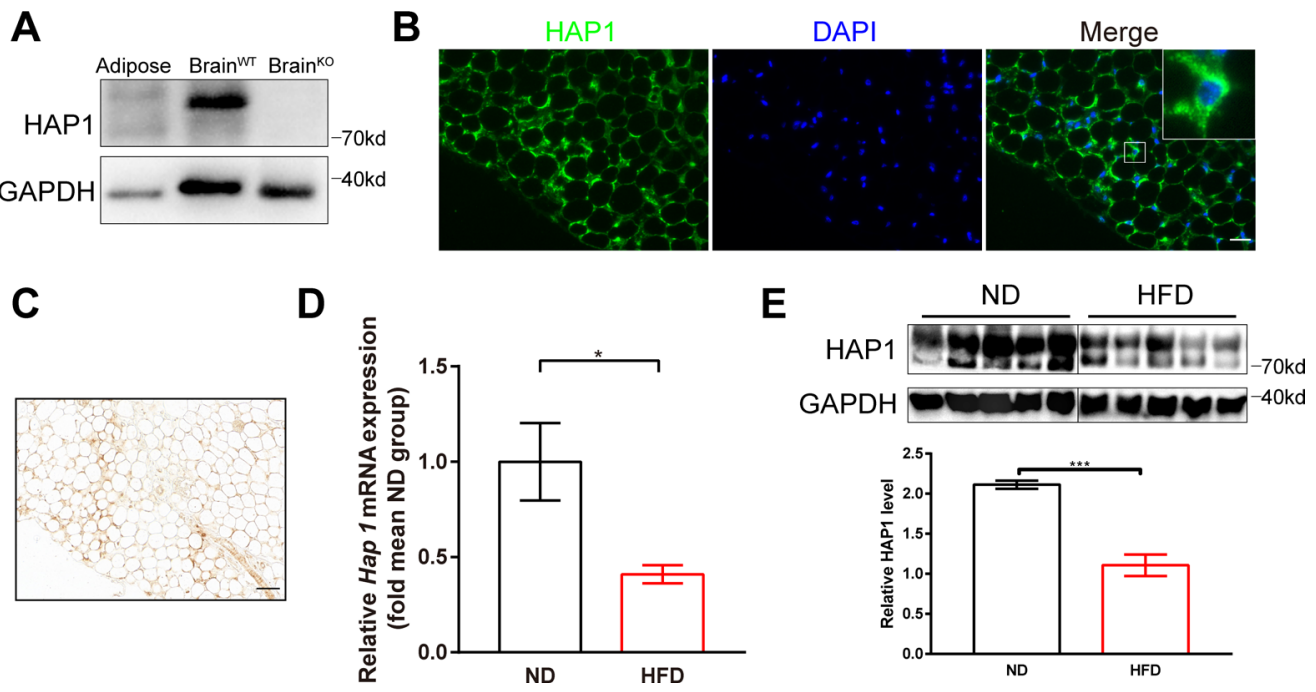

Figure 1 Decreased expression of huntingtin-associated protein 1 (HAP1) in high-fat diet (HFD)-induced diabetic mice. (A) Mouse adipose and brain tissues were harvested for detection of HAP1 by western blotting. Sample from wild-type (WT) brain was used as a positive control, and sample from Hap $1^{-/-}$brain was used as a negative control. (B) Immunofluorescence staining of adipose tissue for HAP1 (green). Scale bar, $25 \mu \mathrm{m}$. (C) Immunohistochemistry staining of adipose tissue for HAP1. Scale bar, $50 \mu \mathrm{m}$. (D and E) Adipose tissues were harvested from both HFD and normal diet (ND)-fed mice at 25 weeks. (D) Hap1 mRNA quantification with quantitative PCR. $\beta$-actin was used as the internal reference ( $n=3$ animal per group). (E) HAP1 protein assessed by western blotting. Glyceraldehyde 3-phosphate dehydrogenase (GAPDH) was used as a loading control ( $n=5$ per group). Data are presented as mean \pm SEM. ${ }^{\star} \mathrm{p}<0.05,{ }^{\star \star \star} \mathrm{p}<0.001$ (unpaired two-tailed t tests). DAPI, $4^{\prime}, 6-$ diamidino-2phenylindole.

exhibited an abnormal glucose metabolism phenotype. However, this might be either due to decreased insulin secretion (figure 2C) or due to disturbed insulin response.

To find out which was the possible reason for the abnormal glucose metabolism in $\mathrm{Hapl}^{+/-}$mice, we used the same amount of insulin to stimulate primary adipocytes derived from $\mathrm{HapT}^{--}$and WT mice. Glucose consumption for 24 hours (long-term effect) ${ }^{32}$ and 30 min (instantaneous response to insulin) was measured. Primary $\mathrm{HapI}^{-/}$adipocytes showed lower glucose consumption in response to insulin stimulation compared with WT cells (figure 2F), and the acute insulin-stimulated glucose uptake was also decreased in $\mathrm{HapF}^{-/}$adipocytes (figure 2G). Hence, the insulin response in Hapt adipocytes was attenuated. Taken together, figure 2 data suggested that the deficiency of HAP1 might lead to a delayed glucose response to insulin stimulation and contribute to the development of T2DM in $\mathrm{Hapl}^{+/-}$mice.

\section{GLUT4 translocation was nearly abolished in Hap1 $^{-/-}$ adipocytes}

It is known that GLUT4 is the main insulin-sensitive glucose transporter responsible for glucose uptake. GLUT4 can increase the cellular glucose uptake via translocation of intracellular vesicles to plasma membrane under insulin stimulation. ${ }^{8}$ Since HAP1 is important in vesicle trafficking, we speculate that HAP1 might be involved in the trafficking of GLUT4 vesicles in adipocytes. ${ }^{1011} 1416$ To assess the direct impact of HAP1 on GLUT4 translocation, we stimulated primary adipocytes with insulin and monitored GLUT4 translocation using fluorescence microscopy. As shown in line-scan analysis and quantification result (figure 3A,B), the typical insulin-responsive translocation of GSVs from perinuclear region to plasma membrane was absent in $\mathrm{HapI}^{-}$ - cells during insulin stimulation (analyzed by $\chi^{2}$ test, $\mathrm{p}<0.0001)$. Figures with more cells at low magnification were in online supplemental figure S5 to prove that the results are not unique but common. Furthermore, plasma membrane-bound GLUT4 in $\mathrm{HapF}^{1-}$ cells was not changed before and after insulin stimulation compared with that of WT cells (figure 3C). Taken together, these data suggest that HAP1 may play a direct role in GLUT4 translocation.

\section{HAP1 interacted with GLUT4 in vivo and in vitro}

To investigate whether HAP1 could interact with GLUT4, HEK293 cells were cotransfected with HAP1-CFP and GLUT4-mCherry. There are two isoforms of HAP1: HAP1A and HAP1B. They have same N-terminal sequences and different C-terminal sequences, while the protein-binding domains are on the N-terminal. HAP1A is the main isoform involved in intracellular transport and the most studied isoform at present, so the HAP1 plasmid used here encodes HAP1 isoform A. These two molecules were colocalized in an intracellular location (figure 4A). The similar observation was also confirmed in WT mouse adipose tissue and adipocytes (figure 4A). The Co-IP assays results showed the detection of HAP1 protein in GLUT4 immunoprecipitated samples in HEK293 cells and in vivo adipose tissue (figure $4 \mathrm{~B}$, upper row, lanes 3 and 6 ) and vice versa (figure 4B, lower row, lanes 3 and 6 ), compared with negative control (figure $4 \mathrm{~B}$, lanes 2 


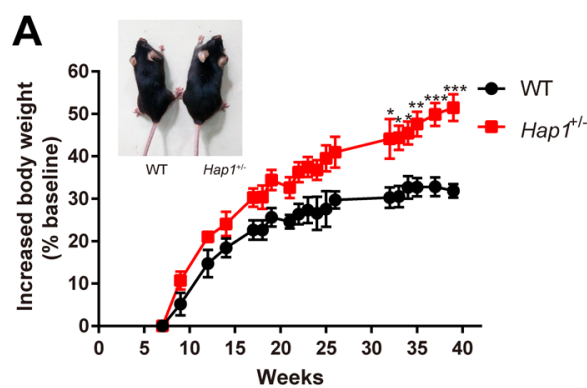

D

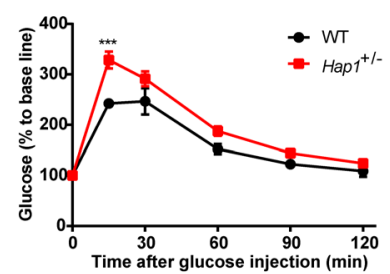

$\mathbf{F}$

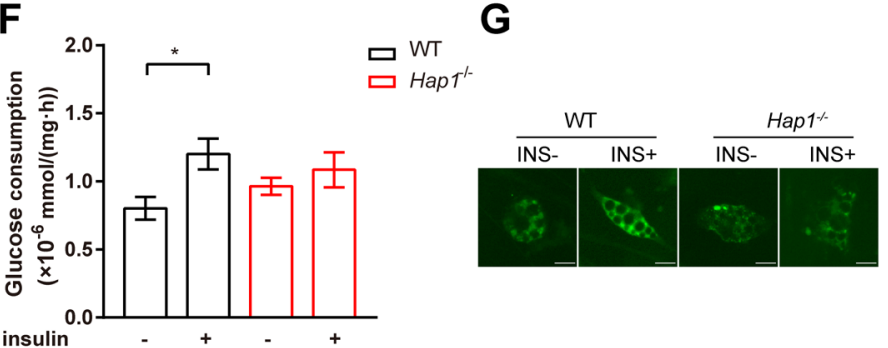

B

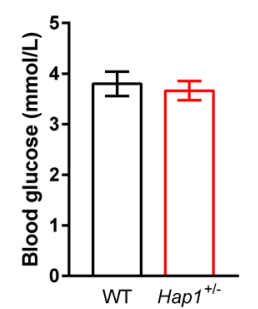

$E$
C

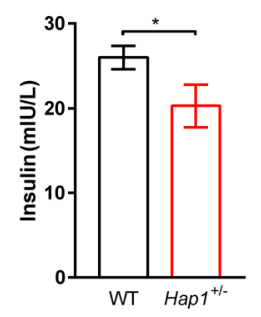

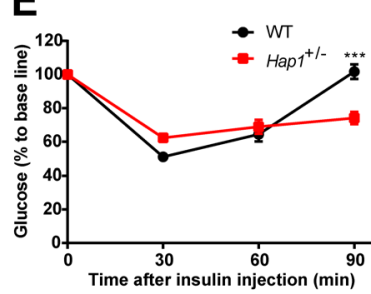
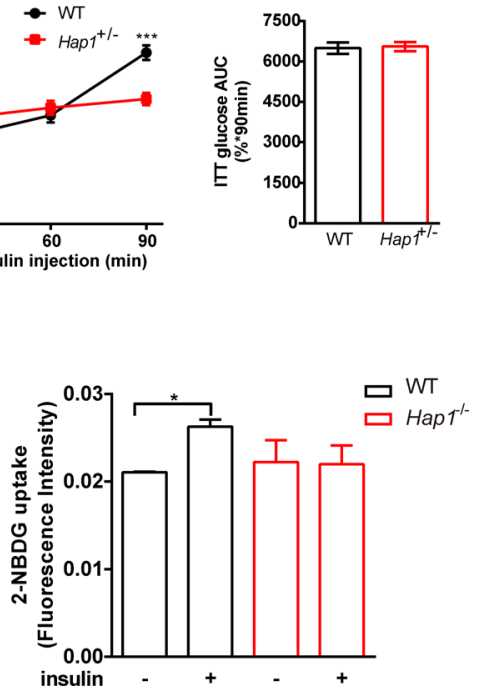

Figure 2 Impaired glucose uptake in $H a p 1^{+/-}$mice as well as in Hap1-knocked out adipocytes. Hap $1^{+/-}$and WT mice were fed with ND, and glucometabolic parameters were monitored. (A) Representative images of $\mathrm{Hap1}^{+/-}$( $32 \mathrm{~g}$ body weight) and WT $(26 \mathrm{~g})$ mice at week 40 and kinetics of body weight increase rate of mice ( $\mathrm{n}=6$ for Hap $1^{+/-}$mice and $\mathrm{n}=4$ for WT mice), $p<0.001$ for genotype effect across all time points (two-way ANOVA). (B) Blood glucose levels of mice fasting for 16 hours at 25 weeks ( $n=7$ for Hap $1^{+/-}$mice and $n=4$ for WT mice), $p=0.2589$. (C) Plasma insulin levels of Hap $1^{+/-} \pm(n=5$ per group), ${ }^{*} \mathrm{p}<0.05$. (D) Relative blood glucose levels and AUC during a GTT at 25 weeks $\left(n=7\right.$ for Hap ${ }^{+/-}$mice and $n=4$ for WT mice), $p=0.0018$ for genotype effect (two-way ANOVA), $p=0.012$ for genotype and time interaction. (E) Relative blood glucose levels and AUC during an ITT at 25 weeks ( $n=6$ for Hap $1^{+-}$mice and $n=4$ for WT mice), $p<0.001$ for genotype and time interaction. (F) Glucose consumption in primary Hap $1^{-/}$and WT adipocytes after 24-hour insulin stimulation ( $n=9$ per group). (G) The representative images and microplate fluorimeter measurement of 2-NBDG uptake of primary Hap $1^{-1 /}$ and WT adipocytes in the absence $(0 \mathrm{nM})$ and presence $(100 \mathrm{nM})$ of insulin for $30 \mathrm{~min}$ (scale bar, $20 \mu \mathrm{m}, \mathrm{n}=3$ per group). Data are presented as mean \pm SEM. ${ }^{*} \mathrm{p}<0.05,{ }^{* *} \mathrm{p}<0.01$ and ${ }^{* \star *} \mathrm{p}<0.001$ (unpaired two-tailed $\mathrm{t}$ tests for bar charts; two-way ANOVA with Bonferroni multiple comparisons test for time plots, for $\mathrm{Hap}^{+/-}$versus WT mice at time point shown). ANOVA, analysis of variance; AUC, area under the curve; GTT, glucose tolerance test; HAP1, huntingtin-associatedprotein 1; INS, insulin; ITT, insulin tolerance test; 2-NBDG, 2-(N-(7-Nitrobenz-2-oxa-1,3-diazol-4-yl)Amino)-2-Deoxyglucose; ND, normal diet; WT, wild type.

and 5) and positive control (figure $4 \mathrm{~B}$, lanes 1 and 4 ). The endogenous expression of untransfected HEK293 cells was tested in figure $4 \mathrm{C}$.

\section{HAP1 formed a protein complex with GLUT4 via sortilin}

Sortilin is one of the components of GSV, which is necessary for GLUT4 vesicle formation, transport and glucose storage. ${ }^{133435}$ Since sortilin has been reported to interact with GLUT4 and HAP1, respectively, ${ }^{9} 1213$ we tested the hypothesis that GLUT4, HAP1 and sortilin could form a complex. We found both HAP1 and GLUT4 were present in the sortilin-immunoprecipitated sample (figure 5A, rows I and II, lane 3), and the presence of sortilin was detected in both HAP1 and GLUT4 immunoprecipitated samples of cotransfected HEK293 cells (figure 5A; rows III and IV, lane 3). To confirm the interaction of the three proteins in vivo, adipose tissue was used for Co-IP assays and showed the similar results (figure 5A, lanes 4-6). These data supported the formation of HAP1, GLUT4 and sortilin complex.

To identify the association among the three proteins, Co-IP assays were performed with single plasmid transfected or sortilin-siRNA/two plasmids cotransfected HEK293 cells. Sortilin was detected in HAP1 or GLUT4 immunoprecipitated samples of HEK293 cells transfected with HAP1 or GLUT4 plasmid, respectively (figure 5C,D). The results showed that sortilin bound with HAP1 and GLUT4, respectively, which is consistent with published data. ${ }^{912}{ }^{13}$ When endogenous sortilin was knocked down by sortilin-siRNA (figure 5B), the association between HAP1 and GLUT4 was nearly abolished 
A
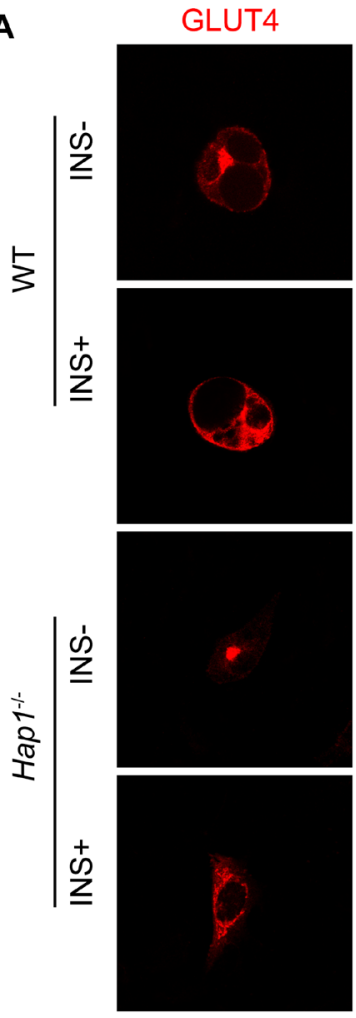

B

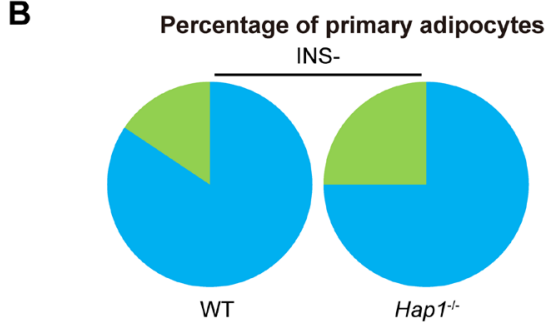

C

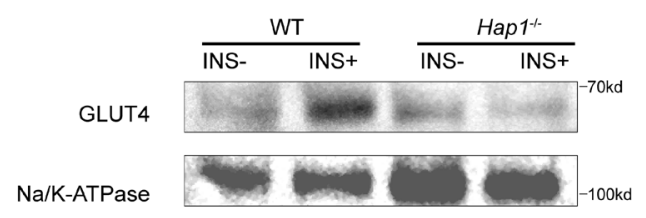

DAPI
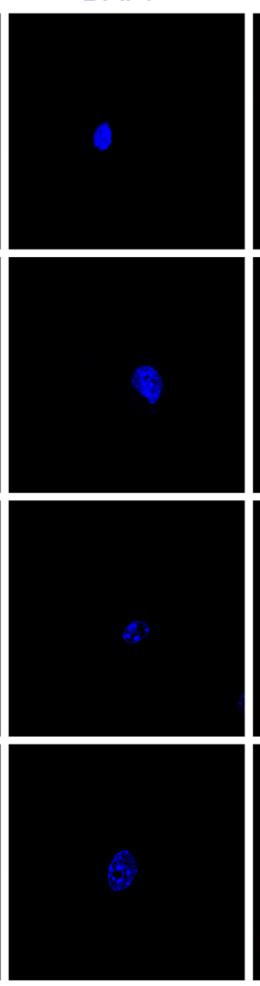
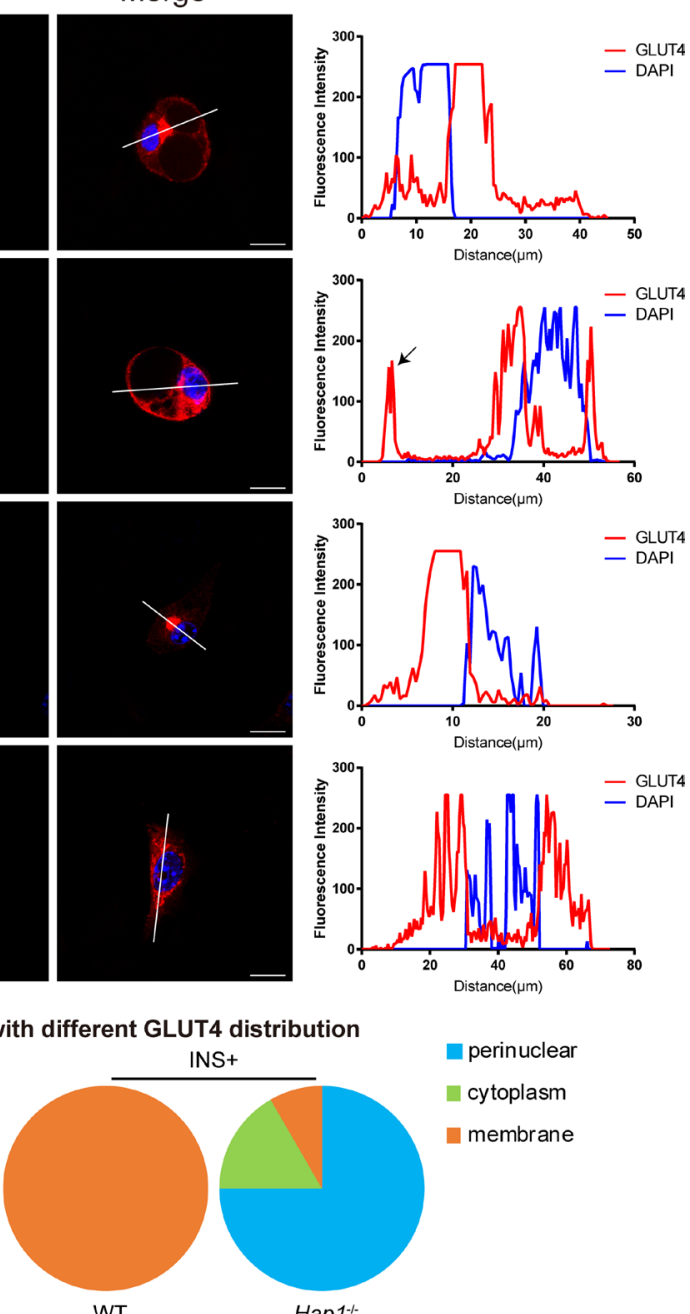

INS+

mperinuclear

cytoplasm

- membrane
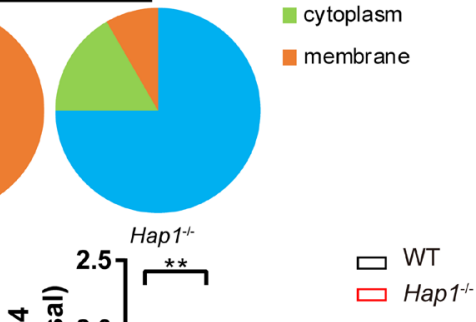

trafficking of the GABA(A) receptor. Decreased expression of mouse hypothalamic HAP1 reduces the level and activity of hypothalamic GABA(A) receptors, leading to declined food intake and body weight. ${ }^{33-38}$ These findings indicate HAP1 may regulate body energy metabolism by regulating neuronal function. However, our study
Prior studies have recorded the importance of HAP1 in feeding behavior. HAP1 is involved in the intracellular

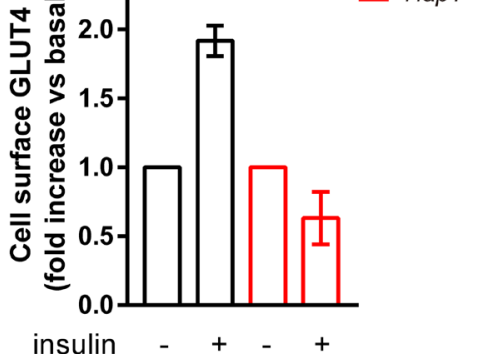

Figure 3 Regulation of glucose transporter isoform 4 (GLUT4) translocation in adipose tissue by huntingtin-associatedprotein 1 (HAP1). (A) Immunofluorescence staining of GLUT4 (left) with cultured primary adipocytes from wild-type (WT) and Hap1 mice after 30 min insulin stimulation following 2-hour starvation and line-scan quantifications for the indicated lines drawn proportion of primary adipocytes with different GLUT4 immunofluorescence distribution at basal state and after 30 min insulin (INS) stimulation ( $n=24$ for Hap $1^{-/-}$cells and $n=56$ for WT cells) $\left(\chi^{2}\right.$ test $\left.p<0.0001\right)$. (C) Detection of membrane-bound GLUT4 in primary $\mathrm{Hap}^{-/-}$and WT adipocytes after $30 \mathrm{~min}$ insulin stimulation following 2-hour starvation by western blotting. Na/KATPase was used as a membrane loading control. Data are presented as mean \pm SEM from three independent experiments.

(figure 5E), which indicates the interaction between HAP1 and GLUT4 was mediated by sortilin.

\section{DISCUSSION}


A
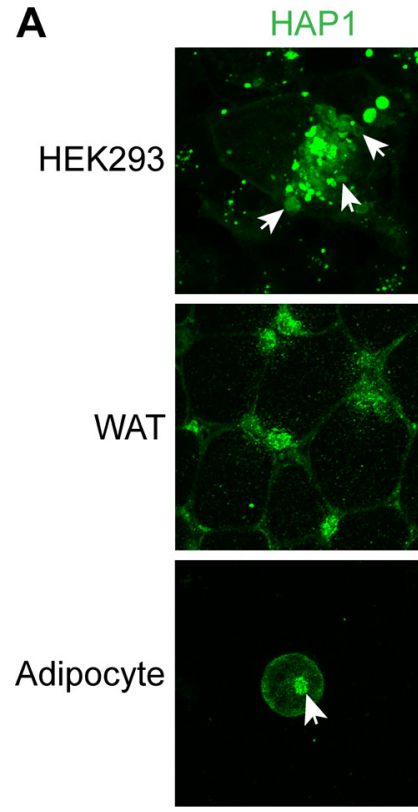

GLUT4
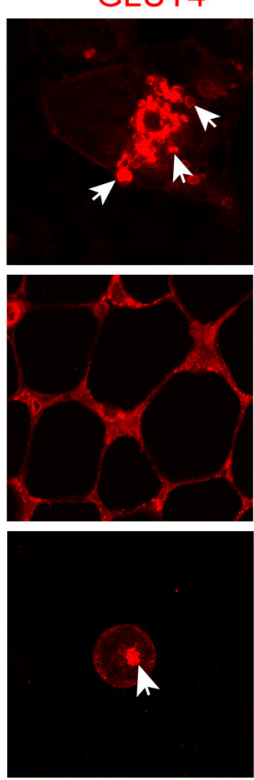

Merge
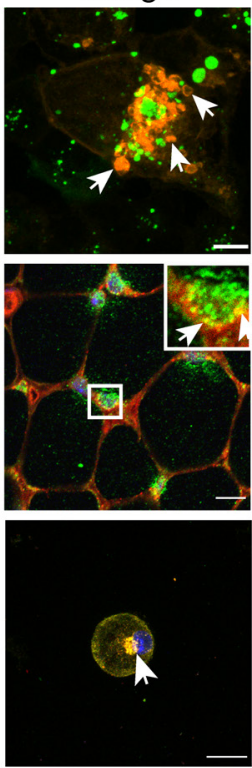

B

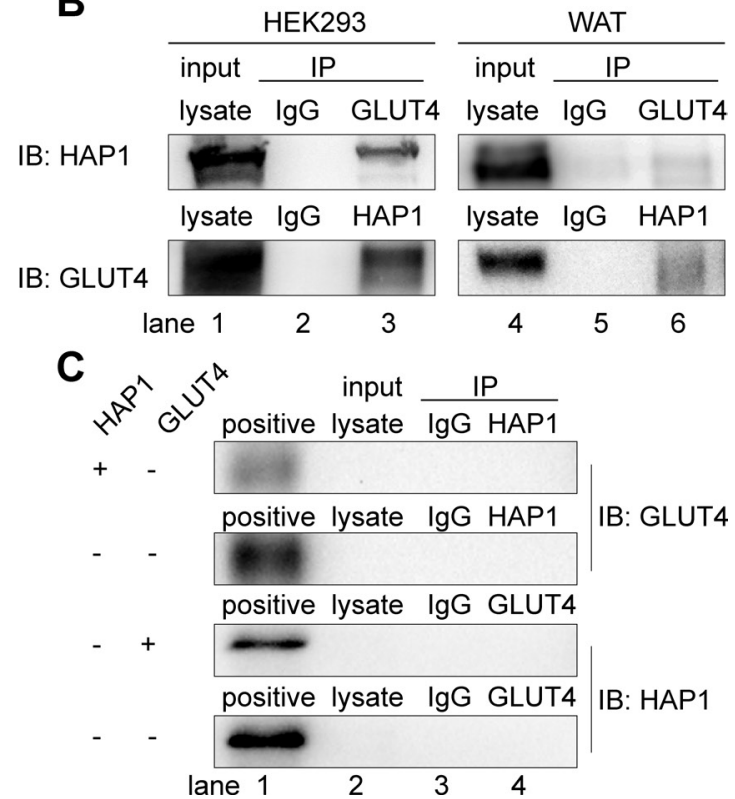

Figure 4 Huntingtin-associatedprotein 1 (HAP1) interacted with glucose transporterisoform 4 (GLUT4) in vivo and in vitro. (A) HAP1 and GLUT4 were colocalized in an intracellular location. Top row: HAP1-CFP and GLUT4-mCherry cotransfected HEK293 cells. Scale bar, $10 \mu \mathrm{m}$. Middle row: Double staining of mouse adipose tissue with HAP1 (green) and GLUT4 (red). Scale bar, $100 \mu \mathrm{m}$. Bottom row: Double staining of wild-type (WT) primary adipocytes with HAP1 (green) and GLUT4 (red). Scale bar, $20 \mu \mathrm{m}$. The nuclei (blue) were stained with 4',6-diamidino-2-phenylindole (DAPI). (B) Co-immunoprecipitation (Co-IP) assay was applied for identification of the interaction between HAP1 and GLUT4. Detection of HAP1 and GLUT4 in HEK293 cells cotransfected with HAP1 and GLUT4 plasmids (left) and mouse adipose tissue (right) by western blotting. Lanes 1 and 4 for the positive control of total protein lysate, lanes 2 and 5 for the IgG as negative control, and lanes 3 and 6 for the Co-IP sample. (C) Co-IP was applied for the identification of endogenous HAP1 and GLUT4 expression of HEK293 cells. HEK293 cells were with or without HAP1 or GLUT4 plasmid, and the other protein was detected by western blotting. Lane 1 for the positive control of HEK293 cells cotransfected with HAP1 and GLUT4 plasmids' total protein lysate, lane 2 for total protein lysate of sample for Co-IP, lane 3 for the IgG as negative control and lane 4 for the Co-IP sample. IB, immunoblotting; IP, immunoprecipitation; WAT, white adipose tissue.

with Hap1 knockdown mice showed higher body weight from week 25. This could not be explained by decreased food intake. On the other hand, the fact that blood insulin levels regulate the expression of hypothalamic and brainstem HAP1, ${ }^{37} 38$ and HAP1 regulates insulin release from pancreatic $\beta$-cells by regulating actin-based internalization of insulin endosomes, ${ }^{1139}$ which is proved by our plasma insulin results. Recently, Shieh et al speculated HAP1 may regulate the intracellular transport of GLUTs in neurons along with motor proteins and be the bridge linking Alzheimer's disease and diabetes. ${ }^{40}$ These all suggest HAP1 may be closely related to the maintenance of glucose homeostasis in vivo, but the mechanism still is complicated. The fasting blood glucose levels in severe insulin secretion defected mice at 24 weeks is much higher than the WT mice, ${ }^{41}$ while the fasting blood glucose levels in the $\mathrm{Hapl}^{+/-}$mice was similar to WT mice. This is indicating that the abnormal glucose metabolism phenotype may not be due to the mild decrease of insulin secretion. The GTT curves of $\beta$-cell-conditional Hap1 ${ }^{+/-}$ mice did not show any significant difference from that of the WT mice, ${ }^{42}$ but global Hap1 knockdown mice in our study showed delayed metabolic rate of high glucose level. The above fact suggests the involvement of Hapl in other tissues in the glucose metabolism. Hap1 ${ }^{+-}$mice exhibited higher blood glucose level compared with WT mice after exogenous insulin injection, a manifestation of insulin resistance, indicating that HAP1 is involved in the process of insulin-mediated glucose uptake. Thus, we proposed a new perspective on the role of HAP1 in body metabolic homeostasis by regulating glucose uptake.

As one of the insulin target tissues, adipose tissue is involved in the development of insulin resistance and is the main culprit of diabetes. ${ }^{43}$ Although the Human Integrated Protein Expression Database suggests that HAP1 may be overexpressed in adipocyte, there was no direct evidence. ${ }^{44}$ Our results demonstrate the expression of HAP1 in adipose tissue for the first time.

As a cytoplasmic transporter, HAP1 interacts with multiple trafficking-related proteins and regulates protein anterograde and retrograde transport in nervous system. ${ }^{45-49}$ One of the interaction proteins of HAP1 in neuron is sortilin. HAP1 forms a protein complex with sortilin and pro-BDNF and prevents pro-BDNF degradation. ${ }^{9}$ Sortilin plays a role in the trafficking of different proteins and participates in vesicle formation, clathrinmediated endocytosis, vesicle transport and sorting in cells. In adipocytes, sortilin is the major component of GSV and acts as a transmembrane scaffold by aggregating other GSV components. ${ }^{12}$ Furthermore, sortilin 


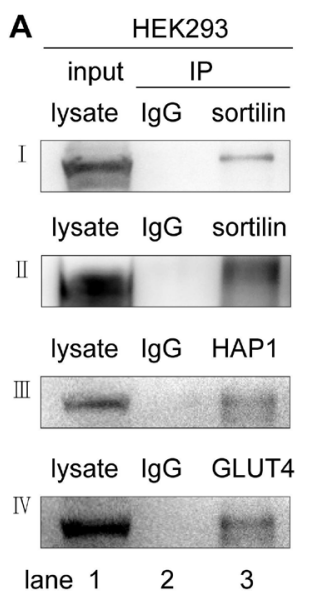

B

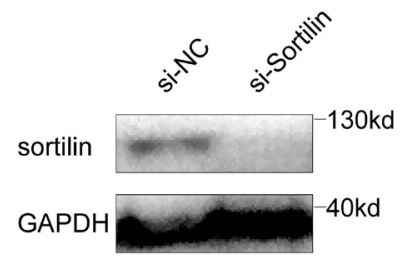

input $\frac{\text { WAT }}{\mathrm{IP}}$

lysate IgG sortilin

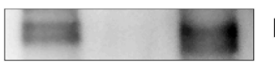

lysate IgG sortilin

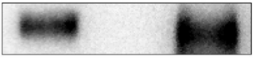

lysate IgG HAP1

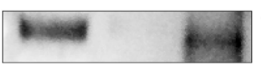

lysate IgG GLUT4

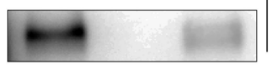

IB: HAP1

IB: GLUT4
C

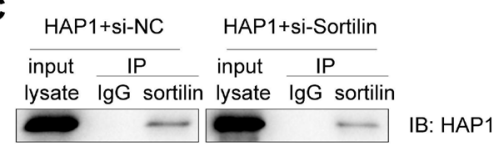

lysate IgG HAP1 lysate IgG HAP1

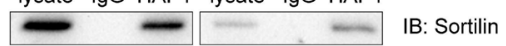

lane $\begin{array}{llllll} & 2 & 3 & 4 & 5 & 6\end{array}$

D GLUT4+si-NC GLUT4+si-Sortilin

input IP input IP

lysate $\lg G$ sortilin lysate $\lg G$ sortilin

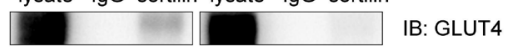

lysate IgG GLUT4 lysate IgG GLUT4

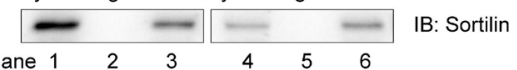

IB: Sortilin participates in the transport of GLUT4 and protects GLUT4 from being sorted into lysosomes for degradation, via binding to the first luminal loop of GLUT4 with its luminal Vps10p domain. ${ }^{13}$ Based on the previous research and our results of HAP1 deficiency in adipocytes leading to a decrease of insulin-stimulated membranebound GLUT4 and the finding of HAP1-sortilin-GLUT4 complex, we hypothesize that HAP1-sortilin-GLUT4 complex may help with GSV stabilization during its translocation.

On the activation of insulin signaling, GSVs in the cytoplasm are transported along the microtubules to the cellular cortex by insulin-regulated kinesin motors (such as KIF5B), where myosin motors (MYO5 and 
MYO1C) continue to transport GSVs to the plasma membrane. ${ }^{14165051}$ With the help of exocyst complex and Soluble N-ethylmaleimide-sensitive Factor Attachment Protein Receptor (SNARE) proteins, the vesicles are tethered to, and then fuse with plasma membrane. ${ }^{52}$ GLUT4 localizes to the cell membrane and exerts physiological functions to transport extracellular glucose into the cells. Any factor affecting GLUT4 translocation may lead to insulin resistance, thus lead to decreased glucose uptake and elevated blood glucose. As the cytoplasmic transporter, HAP1 interacts with KIF5B and MYO5, ${ }^{10} 11$ hence, HAP1 may also participate in the GSVs transport along microtubules and actin.

Taken together, our observation of HAP1-sortilinGLUT4 complex provides new evidence of the involvement of these molecules in GLUT4 translocation stimulated by insulin and explains the correlation between HAP1 and pathogenesis of T2DM. Here we propose a potential mechanism of HAP1 in GLUT4 translocation: HAP1 forms a complex with GLUT4 via sortilin, such complex stabilizes GSVs, while HAP1 also interacts with motor proteins, such as KIF5B and MYO5, to promote the GSV transport along cytoskeleton (figure 5F).

In summary, we provided the firsthand evidence that HAP1 protein is expressed in adipose tissue and HAP1sortilin-GLUT4 complex formation may add one more key mechanism for insulin-stimulated GLUT4-mediated glucose uptake. Our study underlines the important role of adipocyte HAP1 in the pathogenesis of T2DM by regulating GLUT4 translocation.

\section{Author affiliations}

${ }^{1}$ Department of Histology, Embryology and Neurobiology, West China School of Basic Medical Sciences and Forensic Medicine, Sichuan University, Chengdu, Sichuan, China

${ }^{2}$ Institute of Biology, National Institute of Measurement and Testing Technology, Chengdu, Sichuan, China

${ }^{3}$ Department of Pathophysiology, School of Basic Medicine, Shaanxi University of Chinese Medicine, Xianyang, Shaanxi, China

Acknowledgements We thank Professor Qi Peng (King's College London) and Professor Gaoyuan Cao (Rutgers University) for their linguistic assistance during the preparation of this manuscript; Professor Xinfu Zhou (University of South Australia) for his help in study design; Professor Tinghua Wang (Sichuan University) and Professor Jianguo Qi (Sichuan University) for the technical and equipment support.

Contributors YF, G-ZY and XZ conceived the studies; Y-JG, YF, G-ZY and XZ provided critical input to study design, interpretation/discussion of results; $Y-J G$, YF, Y-YC, JZ, WW, Y-YZ, J-RW and XL acquired, analyzed and interpreted data; Y-JG wrote the manuscript. All authors read and approved the final manuscript.

Funding This work was supported by the Office of Science and Technology of Chengdu (grant no: 2016-HM01-00514-SF).

Competing interests None declared.

Patient consent for publication Not required.

Ethics approval All experiments were performed in accordance with the guidelines on the care and use of animals for research purposes by the Institutional Animal Care and Use Committee of Sichuan University, and all the procedures were approved by the Institutional Animal Care and Use Committee of Sichuan University.

Provenance and peer review Not commissioned; externally peer reviewed.

Data availability statement All data relevant to the study are included in the article or uploaded as supplemental information.
Open access This is an open access article distributed in accordance with the Creative Commons Attribution Non Commercial (CC BY-NC 4.0) license, which permits others to distribute, remix, adapt, build upon this work non-commercially, and license their derivative works on different terms, provided the original work is properly cited, appropriate credit is given, any changes made indicated, and the use is non-commercial. See: http://creativecommons.org/licenses/by-nc/4.0/.

ORCID iD

Xue Zhou http://orcid.org/0000-0003-4215-7180

\section{REFERENCES}

1 Czech MP. Insulin action and resistance in obesity and type 2 diabetes. Nat Med 2017;23:804-14

2 Boucher J, Kleinridders A, Kahn CR. Insulin receptor signaling in normal and insulin-resistant states. Cold Spring Harb Perspect Biol 2014;6. doi:10.1101/cshperspect.a009191. [Epub ahead of print: 01 Jan 2014].

3 Zhang W, Shen X-Y, Zhang W-W, et al. Di-(2-ethylhexyl) phthalate could disrupt the insulin signaling pathway in liver of SD rats and L02 cells via PPAR . Toxicol Appl Pharmacol 2017;316:17-26.

4 Takano T, Wu M, Nakamuta S, et al. Discovery of long-range inhibitory signaling to ensure single axon formation. Nat Commun 2017:8:33.

5 Tessneer KL, Jackson RM, Griesel BA, et al. Rab5 activity regulates GLUT4 sorting into insulin-responsive and non-insulin-responsive endosomal compartments: a potential mechanism for development of insulin resistance. Endocrinology 2014;155:3315-28.

6 Garvey WT, Maianu L, Huecksteadt TP, et al. Pretranslational suppression of a glucose transporter protein causes insulin resistance in adipocytes from patients with non-insulin-dependent diabetes mellitus and obesity. J Clin Invest 1991;87:1072-81.

7 Sinha MK, Raineri-Maldonado C, Buchanan C, et al. Adipose tissue glucose transporters in NIDDM. decreased levels of muscle/fat isoform. Diabetes 1991:40:472-7.

8 Stöckli J, Fazakerley DJ, James DE. Glut4 exocytosis. J Cell Sci 2011;124:4147-59.

9 Yang M, Lim Y, Li X, et al. Precursor of brain-derived neurotrophic factor (proBDNF) forms a complex with Huntingtin-associated protein-1 (HAP1) and sortilin that modulates proBDNF trafficking, degradation, and processing. J Biol Chem 2011;286:16272-84.

10 Twelvetrees AE, Yuen EY, Arancibia-Carcamo IL, et al. Delivery of GABAARs to synapses is mediated by HAP1-KIF5 and disrupted by mutant huntingtin. Neuron 2010;65:53-65.

11 Wang Z, Peng T, Wu H, et al. Hap1 helps to regulate actin-based transport of insulin-containing granules in pancreatic $\beta$ cells. Histochem Cell Biol 2015;144:39-48.

12 Shi J, Kandror KV. Sortilin is essential and sufficient for the formation of GLUT4 storage vesicles in 3T3-L1 adipocytes. Dev Cell 2005;9:99-108

13 Pan X, Zaarur N, Singh M, et al. Sortilin and retromer mediate retrograde transport of GLUT4 in 3T3-L1 adipocytes. Mol Biol Cell 2017;28:1667-75.

14 Semiz S, Park JG, Nicoloro SMC, et al. Conventional kinesin KIF5B mediates insulin-stimulated GLUT4 movements on microtubules. Embo J 2003;22:2387-99.

15 Lalioti VS, Vergarajauregui S, Tsuchiya Y, et al. Daxx functions as a scaffold of a protein assembly constituted by GLUT4, JNK1 and KIF5B. J Cell Physiol 2009;218:416-26.

16 Yoshizaki T, Imamura T, Babendure JL, et al. Myosin 5A is an insulinstimulated Akt2 (protein kinase Bbeta) substrate modulating GLUT4 vesicle translocation. Mol Cell Biol 2007;27:5172-83.

17 Li XJ, Li SH, Sharp AH, et al. A Huntingtin-associated protein enriched in brain with implications for pathology. Nature 1995;378:398-402.

$18 \mathrm{Li} \mathrm{XJ}$, Sharp AH, Li SH, et al. Huntingtin-Associated protein (HAP1): discrete neuronal localizations in the brain resemble those of neuronal nitric oxide synthase. Proc Natl Acad Sci U S A 1996;93:4839-44.

19 Liao M, Shen J, Zhang Y, et al. Immunohistochemical localization of Huntingtin-associated protein 1 in endocrine system of the rat. $J$ Histochem Cytochem 2005;53:1517-24.

20 Gutekunst CA, Li SH, Yi H, et al. The cellular and subcellular localization of Huntingtin-associated protein 1 (HAP1): comparison with huntingtin in rat and human. J Neurosci 1998;18:7674-86.

21 Takeshita Y, Fujinaga R, Zhao C, et al. Huntingtin-Associated protein 1 (HAP1) interacts with androgen receptor (AR) and suppresses SBMA-mutant-AR-induced apoptosis. Hum Mol Genet 2006;15:2298-312. 
22 Fujinaga R, Takeshita Y, Yoshioka K, et al. Intracellular colocalization of HAP1/STBs with steroid hormone receptors and its enhancement by a proteasome inhibitor. Exp Cell Res 2011;317:1689-700.

23 Islam MN, Takeshita Y, Yanai A, et al. Immunohistochemical analysis of Huntingtin-associated protein 1 in adult rat spinal cord and its regional relationship with androgen receptor. Neuroscience 2017;340:201-17.

24 Kittler JT, Thomas P, Tretter V, et al. Huntingtin-Associated protein 1 regulates inhibitory synaptic transmission by modulating gammaaminobutyric acid type A receptor membrane trafficking. Proc Nat Acad Sci U S A 2004;101:12736-41.

25 Prigge JR, Schmidt EE. HAP1 can sequester a subset of TBP in cytoplasmic inclusions via specific interaction with the conserved TBP(CORE). BMC Mol Biol 2007;8:76.

26 Kershaw EE, Flier JS. Adipose tissue as an endocrine organ. J Clin Endocrinol Metab 2004;89:2548-56.

27 Galic S, Oakhill JS, Steinberg GR. Adipose tissue as an endocrine organ. Mol Cell Endocrinol 2010;316:129-39.

28 Harwood $\mathrm{HJ}$. The adipocyte as an endocrine organ in the regulation of metabolic homeostasis. Neuropharmacology 2012;63:57-75.

29 Li X, Feng Y, Wu W, et al. Sex differences between APPswePS1dE9 mice in A-beta accumulation and pancreatic islet function during the development of Alzheimer's disease. Lab Anim 2016;50:275-85

30 Yang G-Z, Yang M, Lim Y, et al. Huntingtin associated protein 1 regulates trafficking of the amyloid precursor protein and modulates amyloid beta levels in neurons. J Neurochem 2012;122:1010-22.

31 Yamamoto N, Ueda-Wakagi M, Sato T, et al. Measurement of glucose uptake in cultured cells. Curr Protoc Pharmacol 2015;71:12-14.

32 Yin J, Hu R, Chen M, et al. Effects of berberine on glucose metabolism in vitro. Metabolism 2002:51:1439-43.

33 Li S-H, Yu Z-X, Li C-L, et al. Lack of Huntingtin-associated protein-1 causes neuronal death resembling hypothalamic degeneration in Huntington's disease. J Neurosci 2003;23:6956-64.

34 Bogan JS, Kandror KV. Biogenesis and regulation of insulinresponsive vesicles containing GLUT4. Curr Opin Cell Biol 2010;22:506-12.

35 Bogan JS. Regulation of glucose transporter translocation in health and diabetes. Annu Rev Biochem 2012;81:507-32.

36 Chan EYW, Nasir J, Gutekunst C-A, et al. Targeted disruption of Huntingtin-associated protein-1 (HAP1) results in postnatal death due to depressed feeding behavior. Hum Mol Genet 2002;11:945-59.

37 Sheng G, Chang G-qing, Lin JY, et al. Hypothalamic Huntingtinassociated protein 1 as a mediator of feeding behavior. Nat Med 2006;12:526-33.
38 Niu S-N, Huang Z-B, Wang $\mathrm{H}$, et al. Brainstem Hap1-Ahi1 is involved in insulin-mediated feeding control. FEBS Lett 2011;585:85-91.

39 Pan J-Y, Yuan S, Yu T, et al. Regulation of L-type Ca2+ channel activity and insulin secretion by Huntingtin-associated protein 1. J Biol Chem 2016;291:26352-63.

40 Shieh JC-C, Huang P-T, Lin Y-F. Alzheimer's disease and diabetes: insulin signaling as the bridge linking two pathologies. Mol Neurobiol 2020;57:1966-77.

41 Yagishita Y, Uruno A, Chartoumpekis DV, et al. Nrf2 represses the onset of type 1 diabetes in non-obese diabetic mice. J Endocrinol 2019. doi:10.1530/JOE-18-0355. [Epub ahead of print: 01 Jan 2019].

42 Cape A, Chen X, Wang C-E, et al. Loss of Huntingtin-associated protein 1 impairs insulin secretion from pancreatic $\beta$-cells. Cell Mol Life Sci 2012;69:1305-17.

43 Brandon AE, Liao BM, Diakanastasis B, et al. Protein kinase C epsilon deletion in adipose tissue, but not in liver, improves glucose tolerance. Cell Metab 2019;29:183-91.

44 Fishilevich S, Zimmerman S, Kohn A, et al. Genic insights from integrated human proteomics in GeneCards. Database 2016;2016. doi:10.1093/database/baw030. [Epub ahead of print: $05 \mathrm{Apr}$ 2016].

45 Mackenzie KD, Lim Y, Duffield MD, et al. Huntingtin-Associated protein-1 (HAP1) regulates endocytosis and interacts with multiple trafficking-related proteins. Cell Signal 2017;35:176-87.

46 Rong J, McGuire JR, Fang Z-H, et al. Regulation of intracellular trafficking of Huntingtin-associated protein-1 is critical for TrkA protein levels and neurite outgrowth. J Neurosci 2006;26:6019-30.

$47 \operatorname{Lim}$ Y, Wu LL-Y, Chen S, et al. Hap1 is required for endocytosis and signalling of BDNF and its receptors in neurons. Mol Neurobiol 2018;55:1815-30.

48 Xiang J, Yang H, Zhao T, et al. Huntingtin-Associated protein 1 regulates postnatal neurogenesis and neurotrophin receptor sorting. $J$ Clin Invest 2014:124:85-98.

49 Xiang J, Yan S, Li S-H, et al. Postnatal loss of Hap1 reduces hippocampal neurogenesis and causes adult depressive-like behavior in mice. PLoS Genet 2015;11:e1005175.

50 Yip MF, Ramm G, Larance M, et al. Camkii-Mediated phosphorylation of the myosin motor Myo1c is required for insulin-stimulated GLUT4 translocation in adipocytes. Cell Metab 2008;8:384-98.

51 Bose A, Guilherme A, Robida SI, et al. Glucose transporter recycling in response to insulin is facilitated by myosin Myo1c. Nature 2002;420:821-4.

52 Jahn R, Scheller RH. SNAREs--engines for membrane fusion. Nat Rev Mol Cell Biol 2006;7:631-43. 